\title{
Accurate laser skin perforation technique aimed at promoting bleeding and reducing pain
}

\author{
Han-Chao Chang, Yu-Hsuan Lin* and Kuo-Cheng Huang \\ Instrument Technology Research Center \\ National Applied Research Laboratories, Hsinchu 30076, Taiwan \\ *marklin@narlabs.org.tw
}

Received 27 November 2014

Accepted 22 January 2015

Published 23 February 2015

\begin{abstract}
Laser skin perforation is an effective and promising technique for use in blood collection. In this study, the relation between the perforation profile of skin and laser irradiation at various energies is discussed. Increasing laser energy does not uniformly expand the size and depth of a hole because the shallow depth of field (DOF) of the focused light primarily concentrates energy on the skin surface. In practice, the hole gradually transforms from a semielliptical shape to an upsidedown avocado shape as the laser energy increases. This phenomenon can increase the amount of bleeding and reduce pain. The findings support the feasibility of developing an accurate laser skin perforation method.
\end{abstract}

Keywords: Laser skin perforation; blood collection; Er; YAG laser; diabetic.

\section{Introduction}

Diabetics must check their blood glucose levels daily and endure constant skin perforations, affecting their quality of life; developing a laser skin perforator could yield real benefits for such users. ${ }^{1-3}$ Compared with stainless steel lancets, laser skin perforators inflict relatively low levels of pain, allowing effective blood sampling and rapid wound healing. ${ }^{4}$ When the skin surface is irradiated using short pulses of high power density radiation, skin tissue is locally ablated ${ }^{5-7}$; this forms a small hole, which bleeds if the hole is deep and injures a capillary. Because this laser perforation method involves no contact with the skin, infections can be avoided and this blood sampling technique has numerous useful medical applications. ${ }^{8-10}$

An Er:YAG laser $(2.94 \mu \mathrm{m}$ wavelength $)$ is the most commonly used laser for skin perforation because it emits radiation that is easily absorbed by water molecules within the body. Moreover, this laser penetrates to a more shallow depth compared with Ho:YAG and Nd:YAG lasers, diminishing the resulting pain. ${ }^{11}$ In addition, using short pulses and a focus lens generates smaller wounds compared with those inflicted using traditional lancets. However, excessively small holes may not yield sufficient blood collection. Even if the penetration depth of a hole is adequate and the capillary is open, the flow

*Corresponding author.

This is an Open Access article published by World Scientific Publishing Company. It is distributed under the terms of the Creative Commons Attribution 3.0 (CC-BY) License. Further distribution of this work is permitted, provided the original work is properly cited. 
of blood could be obstructed if the passage or exit is too narrow. To facilitate blood collection, holes must exhibit adequately large openings and sufficient depth. Regarding homogeneous material, increasing the laser energy cannot evenly extend the width and depth of a hole; given the complex composition of organic tissue, the perforation behavior of lasers in this context is extremely complicated.

In this study, an Er:YAG laser with varied energy was used to perforate skin. The holes generated by the laser were observed using an upright optical microscope. By using a high numerical aperture (NA) objective lens, the widths of the holes at various depths were recorded and cross-sectional images were reconstructed. Our analysis demonstrated that the profiles of the holes were not uniformly semicircular or wedge-shaped. Because of the shallow depth of field (DOF) of the laser-focus lens, the light energy was primarily concentrated on the skin surface. As laser energy increased, the expansion speed of the hole was slow in deep regions and fast in shallow regions. Understanding this behavior enables selecting of suitable laser energy to form a hole with a wide opening on the skin surface and a narrow aperture between the epidermis and dermis layers. In other words, an accurate skin perforation method can be achieved, facilitating sufficient blood output and minimal pain. This finding can improve laser skin perforation technology and its applications.

\section{Experimental Setup}

We used an Er:YAG laser in the skin perforation experiment because its emission wavelength $(2.94 \mu \mathrm{m})$ matches the absorption peak of water. Figure 1(a) shows the laser components: a drive circuit, capacitor, and an Er:YAG laser module. The Er:YAG laser module comprised an optical resonator, crystal, and focus lens. The maximal energy of the laser was $350 \mathrm{~mJ}$ and the pulse duration was $250 \mu \mathrm{s}$. Because the holes generated by the laser were extremely small, an optical microscope was used to observe the hole diameters and depths, requiring the preparation of a static sample. In the experiment, a section of a pig ear was used as the in vitro test specimen, as shown in Fig. 1(b); pig ear skin tissue is extremely similar to that of human tissue. ${ }^{12,13}$ The thicknesses of the stratum corneum and living epidermis in the pig ear were approximately 20 and $100 \mu \mathrm{m}$, respectively. Similar to human skin, we determined that blood

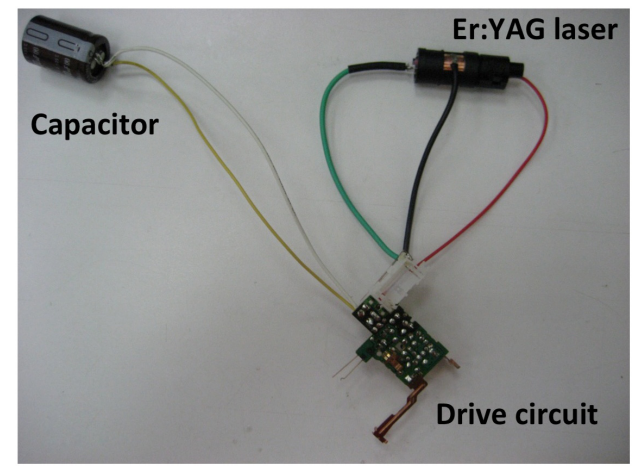

(a)

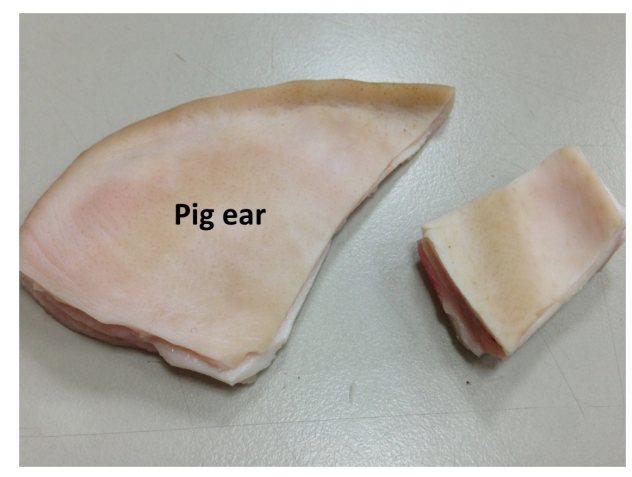

(b)

Fig. 1. Photograph of the (a) laser components and (b) sample of pig ear.

capillaries in the dermis layer of the pig ear opened and began to bleed at a perforation depth of $120-220 \mu \mathrm{m}$.

To diminish pain and limit the amount of bleeding, a focus lens was used to control the laser perforation range. Figure 2(a) illustrates a collimated Er:YAG laser light traveling through a biconvex lens and focusing on the skin of a man's fingertip. The lens was made of transparent plastic; the radius of curvature was $7.85 \mathrm{~mm}$ and the thickness was $3.5 \mathrm{~mm}$. Optical design software (OSOL 6.2.4; Lambda Research) was used to simulate the focusing effect. The theoretical result demonstrated that the effective focal length was approximately $8.87 \mathrm{~mm}$ and the NA was 0.35 . At low NA values, the resolution and DOF of an optical focusing system in the air can be simply expressed as follows:

$$
\begin{aligned}
R & =K_{1} \cdot \frac{\lambda}{\mathrm{NA}}, \\
\mathrm{DOF} & = \pm K_{2} \cdot \frac{\lambda}{(\mathrm{NA})^{2}},
\end{aligned}
$$




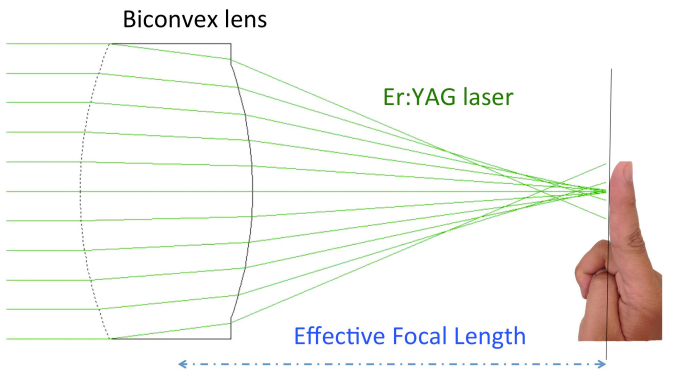

(a)

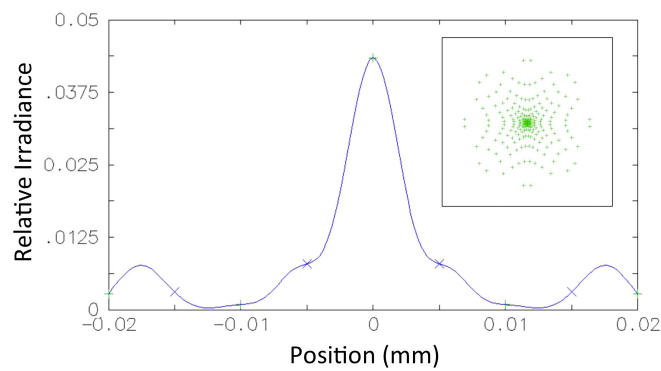

(b)

Fig. 2. (a) Optical simulation of laser skin perforation (not an actual ratio) and (b) irradiance distribution of the focal spot.

where $R$ is the optical resolution; $K_{1}$ and $K_{2}$ are process factors; and $\lambda$ is the wavelength of the light source. Figure 2(b) shows the spatial distribution of relative irradiance of the light spot. The full width at half maximum of the spot was approximately $5 \mu \mathrm{m}$ and the spot size $(13.6 \%$ of max. energy) was approximately $12 \mu \mathrm{m}$. The DOF of the spot was approximately $26 \mu \mathrm{m}$. The Er:YAG laser was unpolarized.

In this study, Er:YAG lasers of three energy levels $(350,250$, and $150 \mathrm{~mJ})$ were used to irradiate the pig skin. A designed opto-mechanical component was employed to determine that the skin surface was located at the focus of the lasers and perpendicular to the optical axis. This component is a hollow plastic cylinder with an outside diameter of $5.2 \mathrm{~mm}$ and inside diameter of $4.4 \mathrm{~mm}$. Based on the effective focal length and lens thickness mentioned above, we set the height of the cylinder at $7.1 \mathrm{~mm}$ in order to facilitate precise control over the distance between the top of the finger and front of the lens. The high energy of the laser causes the skin tissue to vaporize, resulting in partial perforation. A reflective optical microscope (Nikon MM400) with height positioning system was used to measure these perforations. The objective lens was $50 \times$ with a NA of 0.45 . The DOF is shallow enough for observations. Therefore, focal planes were used for optical sectioning to achieve three-dimensional reorganization of these images.

\section{Results and Discussion}

Pig ear epidermis is covered in coarse pores. Thus, the pig ear epidermis pores were first photographed at various focal planes to avoid mistaking them for laser perforations. Figure 3(a) shows a pig ear epidermis pore with a diameter of approximately
$350 \mu \mathrm{m}$ and located on the skin surface. Figure 3(b) shows a pig ear epidermis pore with a diameter of approximately $130 \mu \mathrm{m}$ and located at a depth of $200 \mu \mathrm{m}$ under the skin surface. Figure 3(c) shows the bottom of a pig ear epidermis pore located at a depth of approximately $500 \mu \mathrm{m}$; its size was unclear at this depth. A characteristic feature of pig epidermis pores is their roundness at various depths. Irregular shapes were not observed, and their sizes were extremely consistent. Thus, they can easily be distinguished from laser perforations.

First, an Er:YAG laser with energy of $350 \mathrm{~mJ}$ was used to perforate the skin. Figure 4(a) shows that an irregular hole in the shape of a shallow volcano is formed on the skin surface. Figure 4(b) shows the image presented in Fig. 4(a) after undergoing uniform image processing (the threshold function of ImageJ software was used to filter the background and define the hole size more precisely). Because the hole was not round, only the transverse and longitudinal diameters were measured at 469 and $427 \mu \mathrm{m}$, respectively. This estimation method may lead to deviation from uncertainty or human error associated with the identification of hole boundaries. We repeatedly sampled the diameter of the hole along a single axis and the results presented a difference of less than $5 \%$ among more than 10 individual measurements. We believe that this degree of fluctuation is acceptable. Next, optical images and hole sizes were recorded and measured at various depths, with one image taken at $50-\mu \mathrm{m}$ intervals until the bottom was reached. With this laser energy, the depth of perforation was approximately $250 \mu \mathrm{m}$. Thus, a total of six images with accompanying data were obtained. The significance of these data is analyzed below. Figure 4(c) shows the optical image of the hole bottom, in which sufficient reflected light and a flat base structure can be 

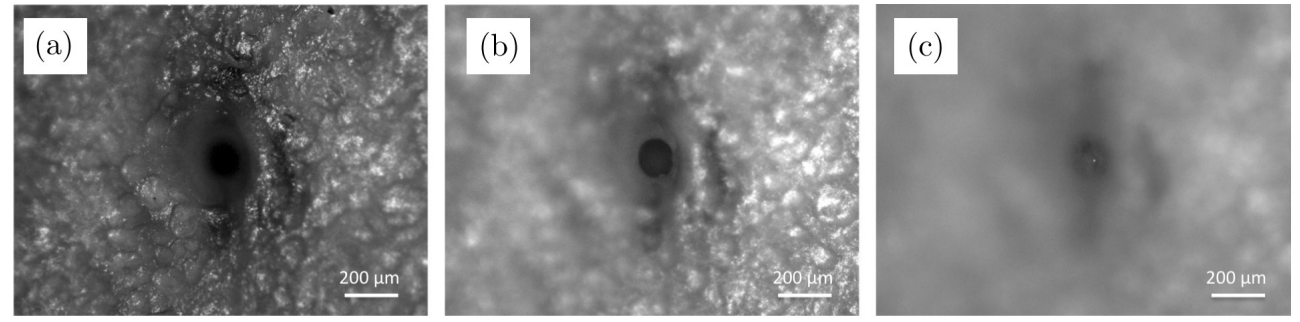

Fig. 3. Photograph of the pig ear epidermis pores at various focal planes.

clearly observed. Figure 4(d) shows the image presented in Fig. 4(c) after undergoing uniform image processing. The contour was elliptical, and the longitudinal and transverse diameters of the hole bottom were 84 and $163 \mu \mathrm{m}$, respectively. Although the laser spot was circular, the skin tissue was not a homogeneous material. Thus, the uniformity of the tissue vaporization varied, especially at greater skin depths.

As shown in Fig. 5, similar methods were employed to measure the skin hole formed by the laser with energy of $250 \mathrm{~mJ}$. Because this energy was less than $350 \mathrm{~mJ}$, the hole depth, approximately $200 \mu \mathrm{m}$, was comparatively shallower. Optical images and hole sizes were recorded and measured at various focal planes, with one image taken at $50-\mu \mathrm{m}$ intervals until the bottom was reached. Thus, a total of five images with accompanying data were obtained. Figure 5(a) shows the optical image of a hole on the skin surface. Figure 5(b) shows the image presented in Fig. 5(a) after undergoing uniform image processing. The longitudinal and transverse diameters of the hole measured 450 and $434 \mu \mathrm{m}$, respectively. Figure 5(c) shows the optical image of the hole bottom. Figure 5(d) shows the image presented in Fig. 5(c) after undergoing uniform image processing. The longitudinal and transverse diameters of the hole bottom measured $74 \mu \mathrm{m}$ and $64 \mu \mathrm{m}$, respectively. Because the pig skin sample was not transparent, irregular hole sidewalls also caused varying amounts of scattering. Thus, optical images taken at different focal planes exhibited differing and unpredictable luminosity at the same level of
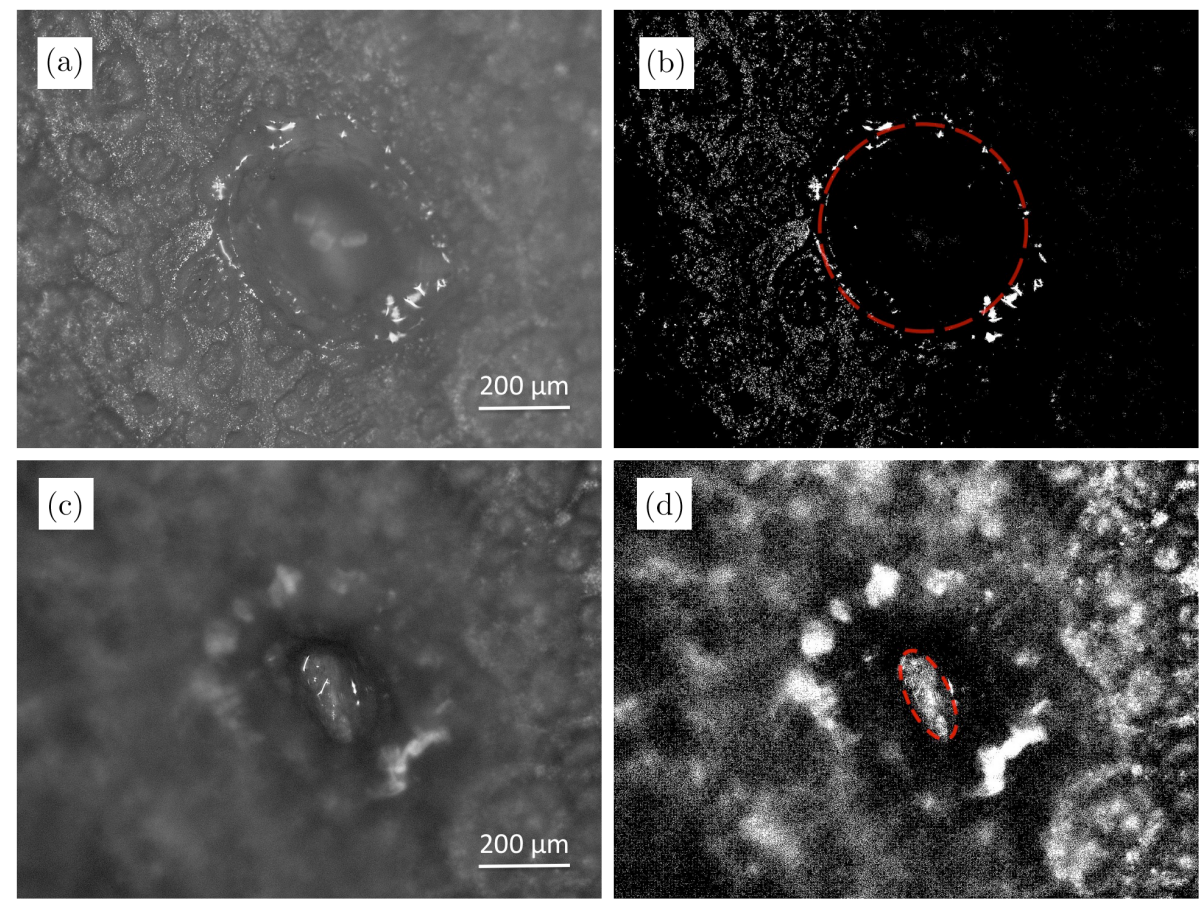

Fig. 4. Skin perforation by Er:YAG laser with energy of $350 \mathrm{~mJ}$. Optical image of the (a) hole top and (c) hole bottom, (b) and (d) are the post-processed images of (a) and (c), respectively. The red dashed ellipse indicates the estimated contour of the hole. 

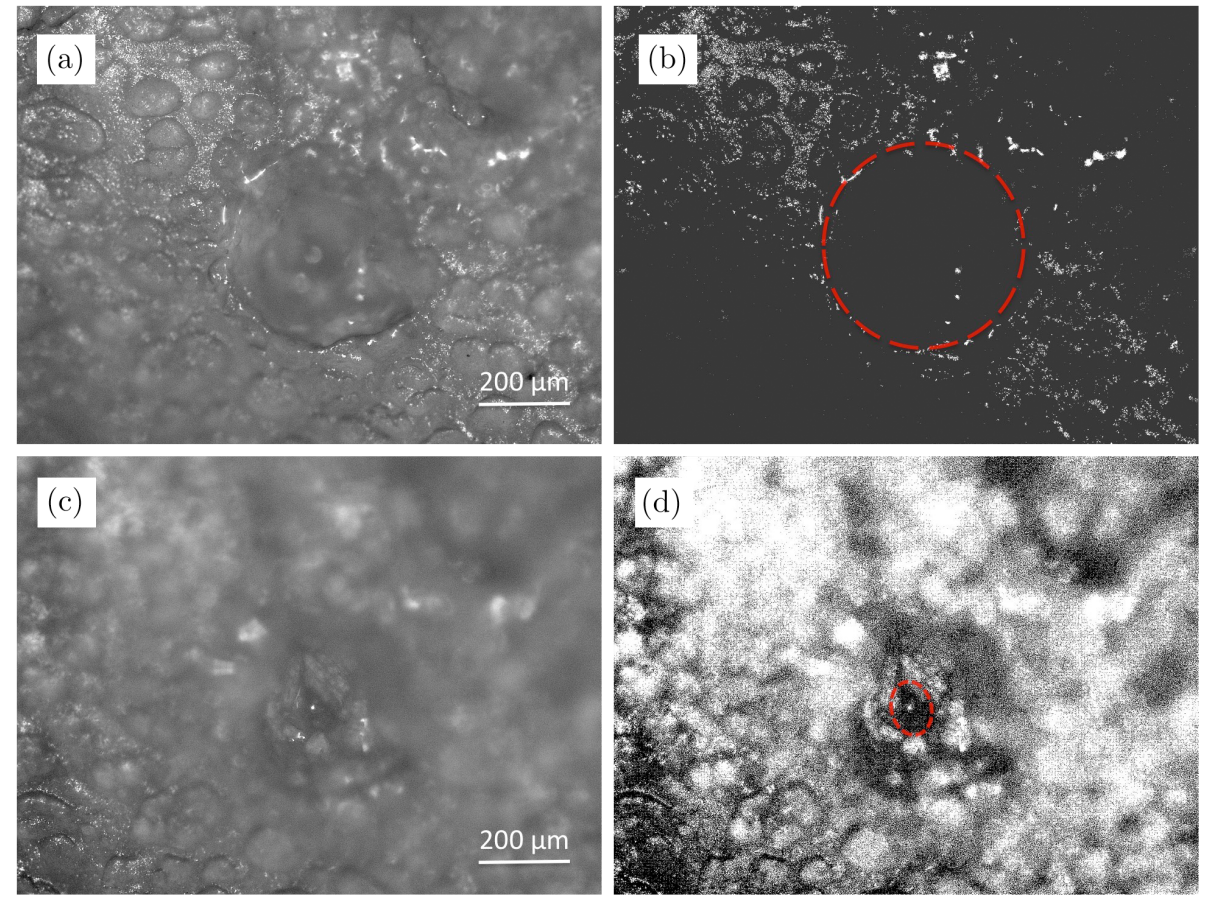

Fig. 5. Skin perforation by Er:YAG laser with energy of $250 \mathrm{~mJ}$. Optical image of the (a) hole top and (c) hole bottom, (b) and (d) are the post-processed images of (a) and (c), respectively. The red dashed ellipse indicates the estimated contour of the hole.

illumination. However, this did not influence the observations of hole size.

Figure 6 shows the perforation created using the laser with energy of $150 \mathrm{~mJ}$. The depth of this hole was approximately $150 \mu \mathrm{m}$. Optical images and hole sizes were recorded and measured at various focal planes, with one image taken at $50-\mu \mathrm{m}$ intervals until the bottom was reached. Thus, a total of four
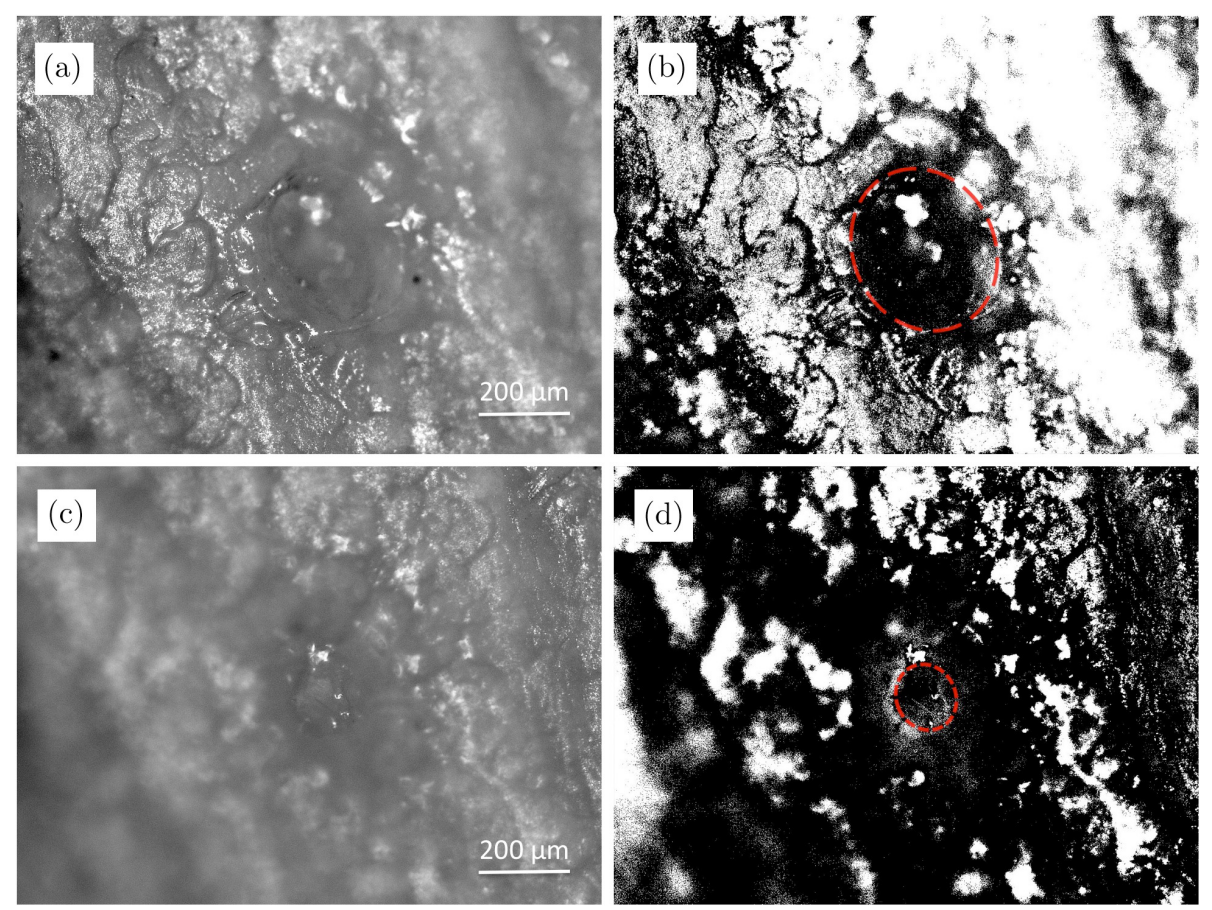

Fig. 6. Skin perforation by Er:YAG laser with energy of $150 \mathrm{~mJ}$. Optical image of the (a) hole top and (c) hole bottom, (b) and (d) are the post-processed images of (a) and (c), respectively. The red dashed ellipse indicates the estimated contour of the hole. 
images with accompanying data were obtained. Figure 6(a) shows the optical image of a hole on the skin surface. Figure 6(b) shows the image presented in Fig. 6(a) after undergoing uniform image processing. The longitudinal and transverse diameters measured $329 \mu \mathrm{m}$ and $342 \mu \mathrm{m}$, respectively. Figure 6(c) shows the optical image of the hole bottom. Figure $6(\mathrm{~d})$ shows the image presented in Fig. 6(c) after undergoing uniform image processing. The longitudinal and transverse diameters of the hole bottom measured $100 \mu \mathrm{m}$ and $101 \mu \mathrm{m}$, respectively. The pig skin surface was not completely flat and contained slight deformations. The height of the samples was manually adjusted in order to observe the associated tilt and deformation. A comparison of the clarity along various focal planes of each area made it possible to record and later analyze the minimum and maximum deviations. Our results revealed that the optical images in Figs. 4-6 possess errors in height ranging between approximately $5 \%$ and $15 \%$.

The diameter values measured at various depths were reorganized to draw sectional views of the holes, as shown in Fig. 7. Figure 7(a) shows the transverse sectional view, and Fig. 7(b) shows the longitudinal sectional view. Curves A, B, and C in the figures represent the silhouettes of the holes formed on the skin by the lasers with energy of 350 , 250 , and $150 \mathrm{~mJ}$, respectively. These two figures show the dimensional relationships at various depths for holes formed through irradiation using varying laser energy. The hole depth was comparatively shallow under relatively low laser energy (Curve C). The uneven tissue only posed little influence on the laser when it penetrated the skin. Thus, the bottom of the hole it formed exhibited excellent symmetry. This means that the transverse and longitudinal sectional views were relatively similar. However, this symmetry gradually declined when the laser energy was increased (Curve B). The amount of uneven tissue that the light was required to penetrate also increased. Therefore, the shape of the hole bottom became irregular. In addition, the deeper region of the hole is distant from the DOF of the focused laser. Thus, the nonlinearity of the energy declined, causing the hole to form the shape of an upside-down avocado. Finally, when the laser energy was further increased (Curve A), the shape of the hole bottom became even more unpredictable. At this time, the capillary blood, nerves, and receptors in the dermis damaged the uniformity of the light, resulting in severe hole deformation.

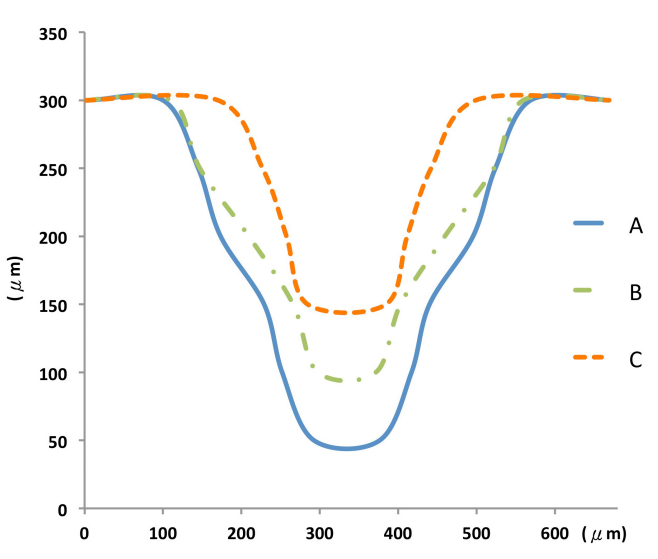

(a)

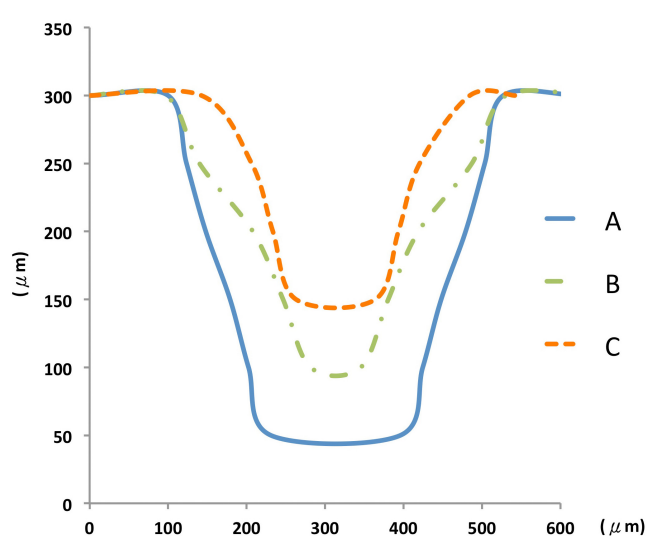

(b)

Fig. 7. (a) The transverse sectional view and (b) longitudinal sectional view of the skin perforation for various laser perforating energies.

Figure 8 shows the relationship between hole width and depth for various laser energies. We measured the transverse and longitudinal diameters of the holes at different depths and then used the average value as the width value in Fig. 8. Curves

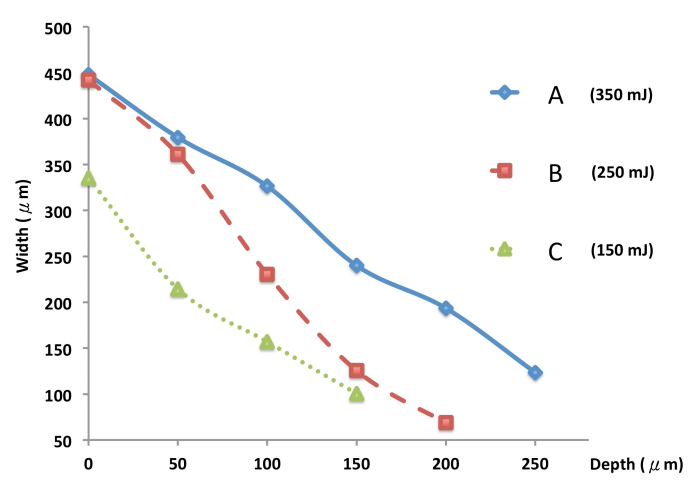

Fig. 8. Illustration of the hole width versus the hole depth for various laser-perforating energies. 
A, B, and C represent lasers with energy of 350, 250, and $150 \mathrm{~mJ}$, respectively. The data in Fig. 8 show that the sizes of perforations on the skin surface (depth of $0 \mu \mathrm{m}$ ) for Curves A and B did not differ considerably. However, at a depth of $150 \mu \mathrm{m}$, the hole width of Curve B was approximately $114.5 \mu \mathrm{m}$ less than that of Curve A. At a depth of $200 \mu \mathrm{m}$, the hole width of Curve B was approximately $124.5 \mu \mathrm{m}$ less than that of Curve A. The sizes of perforations on the skin surface in Curves B and C differed by approximately $106.5 \mu \mathrm{m}$. However, at a depth of $150 \mu \mathrm{m}$, the hole width of Curve B was only $25 \mu \mathrm{m}$ greater than that of Curve C. These phenomena facilitate the determination of appropriate laser energies for collecting blood from various types of skin. In the past, smaller laser perforations, as represented by Curve $\mathrm{C}$, were considered superior (with sufficient depth conditions) for reducing the long-term pain and discomfort caused by regular blood sampling (such as for diabetic patients). However, excessively small perforations actually prevent sufficient amounts of blood from being extracted smoothly. Superior laser perforation should result in larger holes on the skin surface, and smaller damage area of capillary and nerve within the holes, as demonstrated by the behavior of Curve B. Accordingly, bleeding efficiency can be increased and pain and discomfort reduced.

\section{Conclusion}

In this study, laser skin perforations were performed on pig ear skin by using a focused Er:YAG laser at three energy levels - 150, 250, and $350 \mathrm{~mJ}$. The corresponding hole shapes were observed and analyzed. During the theoretical calculation, the spot size and DOF of the focusing laser were approximately 12 and $26 \mu \mathrm{m}$, respectively. An optical microscope equipped with a high-N.A objective lens was used to perform optical sectioning. Cross-sectional images of the holes were acquired using numerical restructuring. The experimental results showed that deep regions of the hole cross section tended to be asymmetric because the complex skin tissue gradually decreased the uniformity of the laser energy. In addition, the increasing laser energy unevenly expanded the size and depth of the hole because the shallow DOF of the focusing light primarily concentrated energy on the skin surface. This uneven intensity distribution caused the expansion speed of the hole to slow more in the deep regions compared with the shallow regions. Furthermore, we speculate that a focus lens with a higher N.A. value can expand the speed difference. Our findings indicate that accurate skin perforation, allowing sufficient blood output and a minimal amount of pain, can be achieved by selecting a suitable light power and NA for the focus lens. However, these parameters must be individually adjusted according to the skin thickness of the patient. The bio-optical exploration of laser skin perforation behaviors presented in the current study should provide valuable insight to the development of this technology.

\section{References}

1. M. Burge, D. Costello, S. Peacock, N. Friedman, "Use of a laser skin perforator for determination of capillary blood glucose yields reliable results and high patient acceptability," Diabetes Care 21, 871873 (1998).

2. V. Ashok, A. Nirmalkumar, N. Jeyashanthi, "A novel method for blood glucose measurement by noninvasive technique using laser," Int. J. Biol. Life Sci. 6, 127-133 (2010).

3. D. Savastru, E. Ristici, M. Mustata, S. Miclos, M. Rusu, C. Radu, V. Savu, "An Er:YAG blood sampling skin puncher," Proc. SPIE, Vol. 6785 (2007).

4. Z.-C. Wu, G.-Y. Jin, X.-C. Tan, M. Ling, Z. Liang, "Design of Er:YAG laser blood-sampling device," Proc. SPIE, Vol. 7382 (2009).

5. J. Walsh, T. Flotte, T. Deutsch, "Er:YAG laser ablation of tissue: Effect of pulse duration and tissue type on thermal damage," Lasers Surg. Med. 9, 314-326 (1989).

6. R. Hibst, R. Kaufmann, "Effects of laser parameters on pulsed Er:YAG laser skin ablation," Lasers Med. Sci. 6(4), 391-397 (1991).

7. I. Apitz, A. Vogel, "Material ejection in nanosecond Er:YAG laser ablation of water, liver, and skin," Appl. Phys. A 81(2), 329-338 (2005).

8. M. Perez, D. Bank, D. Silvers, "Face with the Erbium:YAG laser," Dermatol. Surg. 24, 653-659 (1998).

9. M. Trelles, M. Ve'lez, S. Mordon, "Correlation of histological findings of single session Er:YAG skin fractional resurfacing with various passes and energies and the possible clinical implications," Lasers Surg. Med. 40, 174-177 (2008).

10. M. Bodendorf, S. Grunewald, T. Wetzig, J. Simon, U. Paasch, "Fractional laser skin therapy," J. der 
Deutschen Dermatologischen Gesellschaft 7, 301308 (2008).

11. J. Cabrera, L. Ponce, M. Arronte, T. Flores, B. Lambert, A. Peña, "Er:YAG laser device for taking blood samples," J. Biol. Sci. 8, 15-18 (2008).

12. L. J. van den Broek, F. B. Niessen, R. J. Scheper, S. Gibbs, "Development, validation, and testing of a human tissue engineered hypertrophic scar model," ALTEX 29, 389-402 (2012).

13. B. Godin, E. Touitou, "Transdermal skin delivery: Predictions for humans from in vivo, ex vivo and animal models," Adv. Drug Deliv. Rev. 59, 11521161 (2007). 